\title{
CCL13 is a Promising Diagnostic Marker for Systemic Sclerosis
}

Koichi Yanaba, M.D., Ph.D.* §, Ayumi Yoshizaki, M.D.* §, Eiji Muroi, M.D., Ph.D.* , Toshihide Hara, M.D., Ph.D.* , Fumihide Ogawa, M.D., Ph.D.* , Kazuhiro Shimizu, M.D., Ph.D.* , Minoru Hasegawa, M.D., Ph.D. ${ }^{\dagger}$, Manabu Fujimoto, M.D., Ph.D. ${ }^{\dagger}$, Kazuhiko Takehara, M.D., Ph.D. ${ }^{\dagger}$ and Shinichi Sato, M.D., Ph.D.* *

* Department of Dermatology, Nagasaki University Graduate School of Biomedical

Sciences, Nagasaki, Japan; ${ }^{\dagger}$ Department of Dermatology, Kanazawa University Graduate School of Medical Science, Kanazawa, Japan; and Department of Dermatology, Faculty of Medicine, University of Tokyo, Tokyo, Japan.

${ }^{\S}$ These authors contributed equally to this work.

Running title: CCL13 levels in SSc

Address correspondence and reprint requests to: Shinichi Sato, M.D., Ph.D., Department of Dermatology, Nagasaki University Graduate School of Biomedical Sciences, 1-7-1 Sakamoto, Nagasaki, 852-8501, Japan.

Phone: 81-95-849-7331

Fax: 81-95-849-7335

E-mail: s-sato@net.nagasaki-u.ac.jp 


\section{Summary}

Objective. To determine serum levels of CCL13 and its clinical associations in patients with systemic sclerosis (SSc).

Methods. Serum CCL13 levels were examined by ELISA in 80 patients with SSc, 20 patients with systemic lupus erythematosus, 20 patients with dermatomyositis, 29 patients with atopic dermatitis, and 50 healthy individuals.

Results. Serum CCL13 levels were elevated in SSc patients $(81.3 \pm 55.8 \mathrm{pg} / \mathrm{ml})$ compared to healthy controls $(15.0 \pm 9.9 \mathrm{pg} / \mathrm{ml} ; \mathrm{p}<0.001)$ and patients with systemic lupus erythematosus $(22.0 \pm 6.9 \mathrm{pg} / \mathrm{ml} ; \mathrm{p}<0.001)$, dermatomyositis $(24.4 \pm 36.1$ $\mathrm{pg} / \mathrm{ml} ; \mathrm{p}<0.001)$, or atopic dermatitis $(18.0 \pm 6.4 \mathrm{pg} / \mathrm{ml} ; \mathrm{p}<0.001)$. Among SSc patients, there were no differences in serum CCL13 levels between limited cutaneous SSc and diffuse cutaneous SSc. In the longitudinal study, CCL13 levels were generally unchanged during the followup.

Conclusion. Serum CCL13 was specifically increased in patients with SSc, but not in patients with systemic lupus erythematosus, dermatomyositis, atopic dermatitis, or healthy controls. CCL13 could be a promising serological marker for SSc.

Key words: systemic sclerosis, chemokine, ELISA, CCL13 


\section{Introduction}

Systemic sclerosis (SSc) is a generalized connective tissue disorder characterized by sclerotic and vascular changes in the skin and various internal organs. A variety of immunological abnormalities of $\mathrm{T}$ and $\mathrm{B}$ lymphocytes have been detected in $\mathrm{SSc}^{1}$. Autoantibodies are positive in $>90 \%$ of patients, and these autoantibodies react to various intracellular components, such as DNA topoisomerase I, centromere, RNA polymerases, U1RNP, and U3RNP ${ }^{2}$. Moreover, hyper- $\gamma$-globulinemia and polyclonal $\mathrm{B}$ cell hyperactivity are detected in patients with $\mathrm{SSc}^{3,4}$. In addition, many previous studies have suggested that some cytokines or growth factors regulate the induction of SSc by stimulating the synthesis of extracellular matrix components, injuring the endothelial cells, and modulating the function of leucocytes ${ }^{1,5}$. In early skin lesions of SSc patients, mononuclear cell infiltration is first seen around small vessels in the dermis $^{4,6}$. Furthermore, the degree of mononuclear cell infiltration correlates with both the degree and progression of skin thickening ${ }^{7}$. Thus, immunological abnormalities and inflammatory cell infiltration play a crucial role in initiating and developing fibrosis in SSc.

Chemokines are the most potent molecules that regulate the migration and recruitment of specific leukocytes. CCL13 (monocyte chemoattract protein-4) is a member of the CC chemokine family and directs the migration of monocytes/macrophages, T-lymphocytes, and eosinophils through its functional ligands CC chemokine receptor (CCR) 2 and $3^{8,9}$. CCL13 is also highly expressed in cartilage from patients with rheumatoid arthritis ${ }^{10}$. Furthermore, CCL13 concentration is increased in sera from patients with rheumatoid arthritis ${ }^{10}$. Therefore, we suggest that CCL13 may have some role in the pathogenesis of SSc. In this study, we examined serum levels of CCL13 in patients with SSc, and related these results to clinical features. In addition, we undertook a retrospective longitudinal study of CCL13 levels in some of these patients to determine the changes in CCL13 over time. 


\section{Materials and Methods}

\section{Patients}

Serum samples were obtained from 80 Japanese patients with SSc (68 females and 12 males). All patients fulfilled criteria for SSc proposed by the American College of Rheumatology $(\mathrm{ACR})^{11}$. These patients were grouped according to the classification system $^{12}$ as follows: 32 patients had limited cutaneous SSc (ISSc) and 48 had diffuse cutaneous SSc (dSSc). Anti-topoisomerase I antibodies (Abs) were positive in 34 patients; anticentromere Abs in 27; anti-RNA polymerase I and III Abs in six; anti-U1RNP Abs in three; anti-U3RNP Abs in one; anti-Th/To Abs in one; and eight were negative. These patients were aged 10-74 years (mean age 50). The mean disease duration was $4.3 \pm 5.6$ (range: $0.2-29$ ) years. At the first visit, seven patients had been treated with low dose steroids (prednisolone 5 to $20 \mathrm{mg} /$ day) and five with low dose D-penicillamine (100 to $500 \mathrm{mg} /$ day). None of the patients had received immunosuppressive treatment.

Twenty patients with systemic lupus erythematosus (SLE) who fulfilled the ACR criteria $^{13}$, 20 with dermatomyositis (DM) who fulfilled the criteria of Bohan and Peter ${ }^{14}$, and 29 patients with atopic dermatitis (AD) who fulfilled the criteria proposed by Hanifin and Rajka ${ }^{15}$ acted as disease controls. Fifty age- and sex-matched healthy Japanese individuals were used as healthy controls.

In a retrospective longitudinal study, we analyzed 68 serum samples from 24 of 80 patients with SSc who could have been followed longitudinally (22 women and two men). There was no bias in patient selection. The mean followup period was $3.9 \pm$ 1.8 (1.3-7.6) years with 2.8 (2-5) time-points. The mean disease duration was $3.9 \pm$ 5.5 (0.4-13.3) years. Ten patients had dSSc and 14 had 1SSc; Anti-topo I Abs were positive in nine patients (all dSSc); and anticentromere Abs in 15 (one dSSc, 14 lSSc). These patients were aged 34-72 years (mean age 56).

Fresh venous blood samples were centrifuged shortly after clot formation. All samples were stored at $-70^{\circ} \mathrm{C}$ before use. 


\section{Clinical assessment}

Complete medical histories, physical examinations, and laboratory tests were conducted for all patients at their first visit, with evaluations especially for pulmonary fibrosis during follow-up visits. Organ system involvement was defined as described $^{16,17}$ : lung = bibasilar fibrosis on chest radiography and high-resolution computed tomography; esophagus = hypomotility shown by barium radiography; heart $=$ pericarditis, congestive heart failure, or arrhythmias requiring treatment; kidney = malignant hypertension and rapidly progressive renal failure with no other explanation; and muscle $=$ proximal muscle weakness and elevated serum creatine kinase. Pulmonary fibrosis was defined as bibasilar interstitial fibrosis on chest high resolution computed tomography. In addition, pulmonary function test, including vital capacity (VC) and diffusion capacity for carbon monoxide (DLco), was also evaluated to examine the severity of pulmonary fibrosis. When the DLco and VC were $<75 \%$ and $<80 \%$, respectively, of the predicted normal values, they were considered to be abnormal. Patients with SSc who were smokers or who had other respiratory disorders that could have affected \%DLco or \%VC were excluded from this study. The modified Rodnan total skin thickness score (TSS) was measured by summing the skin thickness measurements, and determined by palpation on a 0-3 scale in 17 body areas $^{18}$. The protocol was approved by the Nagasaki University Graduate School of Biomedicinal Sciences and Nagasaki University Hospital, and informed consent was obtained from all patients.

\section{Detection of serum CCL13}

Serum CCL13 levels were measured with specific enzyme-linked immunosorbent assay (ELISA) kits (R\&D Systems, Minneapolis, MN, USA), according to the manufacturer's protocol. This ELISA system can detect all circulating CCL13 isoforms. Each sample was tested in duplicate. The detection limit of this assay was $1.92 \mathrm{pg} / \mathrm{ml}$. 


\section{Immunohistochemical staining.}

Skin biopsy samples were taken from the dorsal aspect of the mid-forearm of 6 female patients with SSc (3 dSSc and 3 lSSc) and 6 healthy female volunteers. CCL13 expression in the skin tissue was determined using goat IgG anti-human CCL13 polyclonal Ab (R\&D Systems). Frozen dermal tissues were air-dried and subsequently fixed in cold acetone. The tissue sections were incubated overnight with anti-CCL13 polyclonal $\mathrm{Ab}$ at $4^{\circ} \mathrm{C}$, and then treated with biotinylated rabbit anti-goat IgG $\mathrm{Ab}$ for $45 \mathrm{~min}$ at room temperature, following by incubation with avidin-biotin-peroxidase complex for $30 \mathrm{~min}$. Sections were incubated with diaminobenzidine tetrahydrochloride substrate solution for $5 \mathrm{~min}$, counterstained with methyl green, and embedded in balsam. Isotype-matched control polyclonal Ab was used as a negative control.

\section{Statistical Analysis}

The Mann-Whitney U-test was used to compare CCL13 levels, Fisher's exact probability test to compare frequencies, and Bonferroni's test used for multiple comparisons. Spearman's rank correlation coefficient was used to examine the relationship between two continuous variables. A probability (p) values less than 0.05 were considered statistically significant. 


\section{Results}

\section{Serum CCL13 levels were elevated in patients with SSc}

Serum CCL13 levels at the first visit were significantly higher in patients with SSc $(81.3 \pm 55.8 \mathrm{pg} / \mathrm{ml})$ than healthy controls $(15.0 \pm 9.9 \mathrm{pg} / \mathrm{ml} ; \mathrm{p}<0.001 ;$ Fig. 1$)$, patients with SLE (22.0 $\pm 6.9 \mathrm{pg} / \mathrm{ml} ; \mathrm{p}<0.001)$, patients with DM $(24.4 \pm 36.1 \mathrm{pg} / \mathrm{ml} ; \mathrm{p}<0.001)$, and patients with AD $(18.0 \pm 6.4 \mathrm{pg} / \mathrm{ml} ; \mathrm{p}<0.001)$. Concerning SSc subgroups, CCL13 levels in both patients with dSSc $(82.2 \pm 53.0 \mathrm{pg} / \mathrm{ml})$ and those with $1 S S c$ $(80.0 \pm 60.5 \mathrm{pg} / \mathrm{ml})$ were increased compared with those in healthy controls $(\mathrm{p}<0.001$ and $\mathrm{p}<0.01$, respectively). There was no significant difference in serum CCL13 levels between patients with dSSc and ISSc. Thus, serum CCL13 concentrations were increased in patients with SSc, but not in patients with SLE, DM, AD, or healthy controls.

Clinical and laboratory parameters obtained at the first evaluation were compared between SSc patients with elevated CCL13 levels and those with normal CCL13 levels. Values higher than the mean +2 SD $(34.7 \mathrm{pg} / \mathrm{ml})$ of the control serum samples were considered to be elevated in this study. Serum CCL13 levels did not correlate with clinical features and laboratory data (Table I).

Seventy-six of 80 patients with SSc (46 dSSc, 30 lSSc) exhibited elevated CCL13 levels, while one of 50 healthy controls, one of 20 patients with DM, and one of 29 patients with AD showed increased CCL13 levels (Fig. 1). None of 20 patients with SLE had increased CCL13 concentrations.

\section{Immunohistochemical expression of CCL13 in the skin of patients with SSc.}

Whether CCL13 expression was augmented in the skin of patients with SSc was assessed by immunohistochemical analysis. Patients with SSc had remarkably higher expression of CCL13 in the dermis compared with healthy controls (Fig. 2). Further, fibroblasts in the dermis of patients with SSc exhibited strong CCL13 expression. Thus, augmented CCL13 expression was found in the lesional skin from patients with SSc. 


\section{Longitudinal study of serum CCL13 levels}

To assess changes in serum CCL13 levels over time, 68 serum samples from 24 patients with SSc (10 dSSc and 14 lSSc patients) were analyzed retrospectively. None of these patients had received any treatment at their first visit. Twenty-two of 24 SSc patients exhibited elevated CCL13 levels at their first visit. Serum CCL13 levels in seven of the 22 SSc patients were decreasing during the followup of $2.9 \pm 1.7$ (1.3-7.0) years (Fig. 3a). Their disease duration at the initial visit was $4.0 \pm 4.6$ (0.5-13.0) years. Serum CCL13 levels at the final evaluation were within normal range in one of these patients. During the followup, four of the seven patients exhibited subacute deterioration of interstitial pneumonitis, and three of the four patients received cyclophosphamide pulse therapy (Cases 1-3), and one had steroid pulse therapy (Case 4) for the pulmonary involvement. Treatment with low dose steroids alone was started in two of the remaining four patients.

Serum CCL13 levels in 15 of the SSc patients with high CCL13 levels at first visit remained high during the followup of $4.0 \pm 1.8$ (1.8-7.6) years (Fig. 3b). Their disease duration was $4.8 \pm 6.2(0.4-13.3)$ years. After the initial visit, treatment with low dose steroids alone was started in three of the 15 patients.

Finally, two out of 24 SSc patients examined in this study exhibited normal CCL13 levels at the first visit that stayed within normal range throughout the followup period of $3.9 \pm 1.7$ (2.5-4.7) years (Fig. 3c). Their disease duration was $4.0 \pm$ $4.6(0.5-13.0)$ years. None of these SSc patients had received D-penicillamine or steroids during the followup period. Thus, CCL13 levels were generally unchanged during the followup period, although steroid or immunosuppressive therapy might affect the change. 


\section{Discussion}

This is the first report of elevated serum CCL13 levels in patients with SSc. We assessed patients with SSc, SLE, DM, and AD, but the CCL13 levels were increased only in the patients with SSc (Fig. 1). The CCL13 levels were raised not only in dSSc, but also in ISSc. Elevated CCL13 levels were not associated with clinical features and laboratory data (Table I). Furthermore, the CCL13 levels were generally unchanged during the followup period, although steroid or immunosuppressive therapy might decrease CCL13 concentrations (Fig. 3). Thus, serum CCL13 would be a promising diagnostic marker for SSc.

Although the pathogenesis of SSc remains unclear, it has recently been proposed that oxidative stress may play an important role in the development of $\mathrm{SSc}^{19}$. Ischemia and reperfusion injury following Raynaud's phenomenon can generate reactive oxygen species (ROS) that may result in vascular endothelial damage ${ }^{20,21}$. ROS also stimulate fibroblast proliferation, leading to fibrosis ${ }^{22}$. Interestingly, CCL13 induces the production of $\mathrm{ROS}^{23}$. In this study, CCL13 expression in dermal fibroblasts was similarly augmented in patients with dSSc and ISSc compared with healthy controls (Fig. 2). Serum CCL13 levels were equally elevated in patients with dSSc and ISSc (Fig. 1). Therefere, CCL13 production is likely to be augmented in patients with SSc regardless of the disease severity. Consistently, enhanced oxidative stress in both dSSc and ISSc has been demonstrated since some biomarkers of oxidative stress, including heat shock protein $70, \mathrm{~N}^{\varepsilon}$-(hexanoyl)lysine, are increased in sera from patients with $\mathrm{SSc}^{24,25}$. Thus, CCL13 may play a key role in the pathogenesis of SSc by producing ROS.

Cyclophosphamide and steroid pulse therapy for the interstitial pneumonia seemed to decrease CCL13 levels in sera from patients with SSc (Fig. 3a). Cyclophosphamide treatment improves lung fibrosis and skin thicking associated with $\mathrm{SSc}^{26}$. Therefore, strong immunosuppression may alter systemic inflammatory condition, leading to the improvement of fibrosis to some extent. However, CCL13 expression in bronchoalveolar lavage and lung specimen is substantially enhanced in 
patients with tuberculosis and chronic bronchitis ${ }^{27}$, suggesting that nonspecific inflammation may also induce CCL13 expression. Further studies are needed to address the role of CCL13 in the pathogenesis of SSc. Nonetheless, the measurement of CCL13 in patients who are suspected of SSc may offer an important approach in the diagnosis because no specific serum marker for SSc have been found thus far. 


\section{References}

1 White B. Immunopathogenesis of systemic sclerosis. Rheum Dis Clin Nor Am 1996; 32: 695-708.

2 Okano Y. Antinuclear antibody in systemic sclerosis (scleroderma). Rheum Dis Clin North Am 1996; 22: 709-35.

3 Famularo G, Procopio A, Giacomelli R et al. Soluble interleukin-2 receptor, interleukin-2 and interleukin-4 in sera and supernatants from patients with progressive systemic sclerosis. Clin Exp Immunol 1990; 81: 368-72.

4 Fleischmajer R, Perlish JS, Reeves JRT. Cellular infiltrates in scleroderma skin. Arthritis Rheum 1977; 20: 975-84.

5 Furst DE, Clements PJ. Hypothesis for the pathogenesis of systemic sclerosis. J Rheumatol 1997; 24 (suppl 48): 53-7.

6 Scharffetter K, Lankat-Buttgereit B, Krieg T. Localization of collagen mRNA in normal and scleroderma skin by in-situ hybridization. Eur $J$ Clin Invest 1988; 18: 9-17.

7 Roumm AD, Whiteside TL, Medsger TA, Jr. et al. Lymphocytes in the skin of patients with progressive systemic sclerosis. Quantification, subtyping, and clinical correlations. Arthritis Rheum 1984; 27: 645-53.

8 Uguccioni M, Loetscher P, Forssmann U et al. Monocyte chemotactic protein 4 (MCP-4), a novel structural and functional analogue of MCP-3 and eotaxin. J Exp Med 1996; 183: 2379-84.

9 Garcia-Zepeda EA, Combadiere C, Rothenberg ME et al. Human monocyte chemoattractant protein (MCP)-4 is a novel CC chemokine with activities on monocytes, eosinophils, and basophils induced in allergic and nonallergic inflammation that signals through the CC chemokine receptors (CCR)-2 and -3. J Immunol 1996; 157: 5613-26.

10 Iwamoto $\mathrm{T}$, Okamoto $\mathrm{H}$, Iikuni $\mathrm{N}$ et al. Monocyte chemoattractant protein-4 (MCP-4)/CCL13 is highly expressed in cartilage from patients with rheumatoid arthritis. Rheumatology (Oxford) 2006; 45: 421-4.

11 Subcommittee for Scleroderma Criteria of the American Rheumatism Association Diagnostic and Therapeutic Criteria Committee. Preliminary criteria for the classification of systemic sclerosis (scleroderma). Arthritis Rheum 1980; 23: 581-90.

12 LeRoy EC, Krieg T, Black C et al. Scleroderma (systemic sclerosis): classification, subsets, and pathogenesis. J Rheumatol 1988; 15: 202-5.

13 Tan EM, Cohen AS, Fries JF et al. The 1982 revised criteria for the classification of systemic lupus erythematosus. Arthritis Rheum 1982; 25: 1271-7.

14 Bohan A, Peter JB. Polymyositis and dermatomyositis (first of two parts). $N$ Eng J Med 1975; 292: 344-8.

15 Rajka G, Langeland T. Grading of severity of atopic dermatitis. Acta Derm Venerol 1989; 144: 13-4.

16 Steen VD, Powell DL, Medsger TAJ. Clinical correlations and prognosis based on serum autoantibodies in patients with systemic sclerosis. Arthritis Rheum 1988; 31: 196-203.

17 Sato S, Ihn H, Kikuchi K et al. Antihistone antibodies in systemic sclerosis: association with pulmonary fibrosis. Arthritis Rheum 1994; 37: 391-4. 
18 Clements PJ, Lachenbruch PA, Seibold JR et al. Skin thickness score in systemic sclerosis: an assessment of interobeservar variability in 3 independent studies. J Rheumatol 1993; 20: 1892-6.

19 Murrell DF. A radical proposal for the pathogenesis of scleroderma. $J$ Am Acad Dermatol 1993; 28: 78-85.

20 Suematsu M, Wakabayashi Y, Ishimura Y. Gaseous monoxides: a new class of microvascular regulator in the liver. Cardiovasc Res 1996; 32: 679-86.

21 Butler AR, Flitney FW, Williams DL. NO, nitrosonium ions, nitroxide ions, nitrosothiols and iron-nitrosyls in biology: a chemist's perspective. Trends Pharmacol Sci 1995; 16: 18-22.

22 Murrell GA, Francis MJ, Bromley L. Modulation of fibroblast proliferation by oxygen free radicals. Biochem J 1990; 265: 659-65.

23 Petering $\mathrm{H}$, Hochstetter R, Kimmig D et al. Detection of MCP-4 in dermal fibroblasts and its activation of the respiratory burst in human eosinophils. $J$ Immunol 1998; 160: 555-8.

24 Shimizu K, Ogawa F, Akiyama $\mathrm{Y}$ et al. Increased serum levels of $\mathrm{N}$ (epsilon)-(hexanoyl)lysine, a new marker of oxidative stress, in systemic sclerosis. J Rheumatol 2008; 35: 2214-9.

25 Ogawa F, Shimizu K, Hara T et al. Serum levels of heat shock protein 70, a biomarker of cellular stress, are elevated in patients with systemic sclerosis: association with fibrosis and vascular damage. Clin Exp Rheumatol 2008; 26: 659-62.

26 Tashkin DP, Elashoff R, Clements PJ et al. Cyclophosphamide versus placebo in scleroderma lung disease. $N$ Engl J Med 2006; 354: 2655-66.

27 Miotto D, Christodoulopoulos $\mathrm{P}$, Olivenstein $\mathrm{R}$ et al. Expression of IFN-gamma-inducible protein; monocyte chemotactic proteins 1,3 , and 4; and eotaxin in TH1- and TH2-mediated lung diseases. J Allergy Clin Immunol 2001; 107: 664-70. 


\section{Acknowledgement}

The authors have expressed no conflicts of interest. This work was supported by a grant of Research on Intractable Diseases from the Ministry of Health, Labor and Welfare of Japan. 


\section{Figure Legends}

Fig. 1 Serum levels of CCL13 in patients with SSc, dSSc, ISSc, SLE, DM, AD, and normal controls (Control). Serum CCL13 levels were determined by a specific ELISA. Bars indicate the mean value in each group. The broken line indicates the cut-off value (mean +2 SD of the normal control samples).

Fig. 2 Representative CCL13 expression in normal skin tissues (a) and lesional skin tissues from patients with SSc (b). Original magnification x250.

Fig. 3 Serial changes of serum CCL13 levels during the followup period in SSc patients with elevated CCL13 levels at their first visit but decreasing during followup (a), SSc patients with elevated CCL13 levels at their first visit and stable during followup (b), and SSc patients with normal CCL13 levels at first visit (c). Serum CCL13 levels were determined by a specific ELISA. The broken lines indicate the cut-off value. 


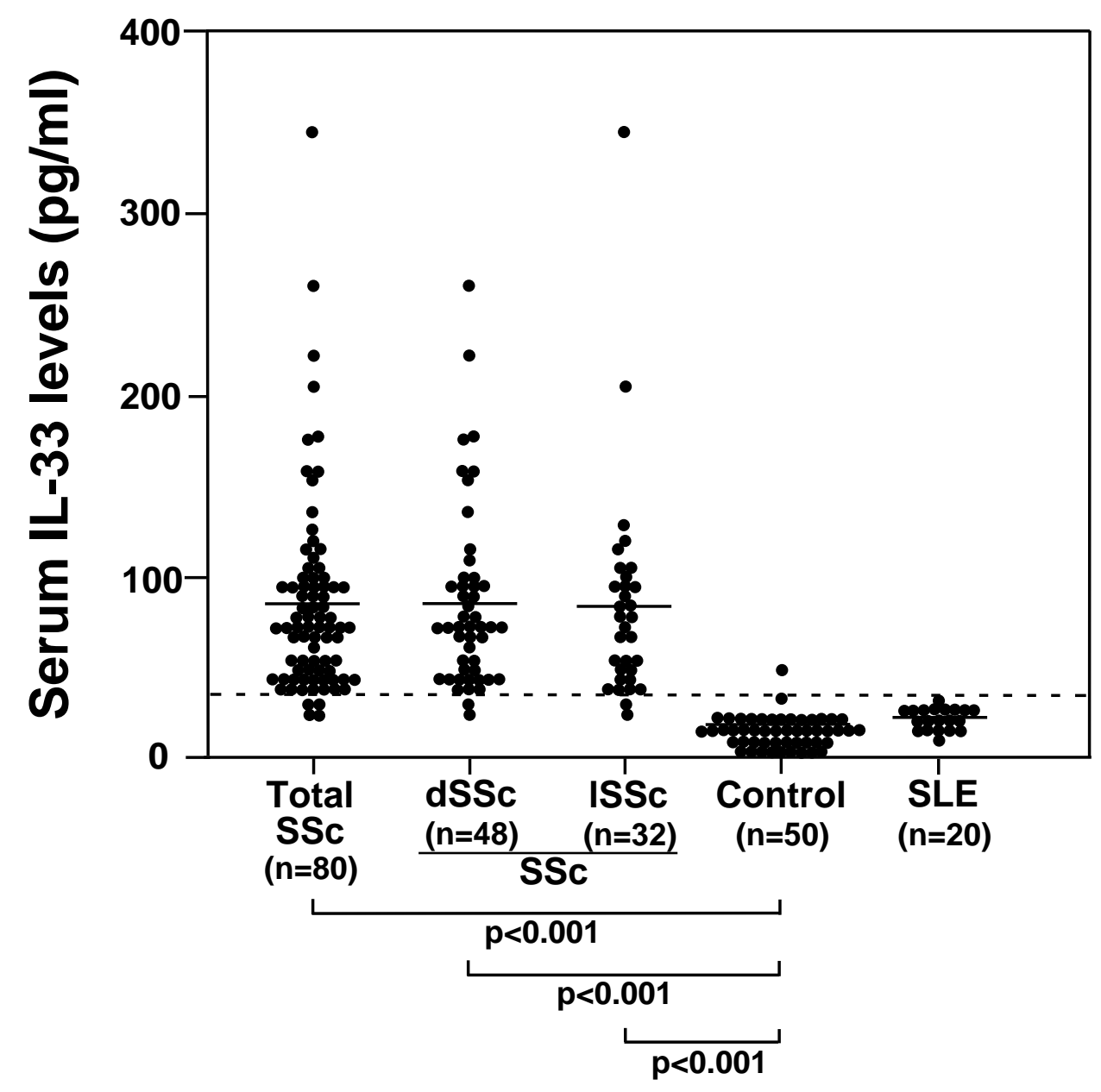

Figure 1

Yanaba et al. 

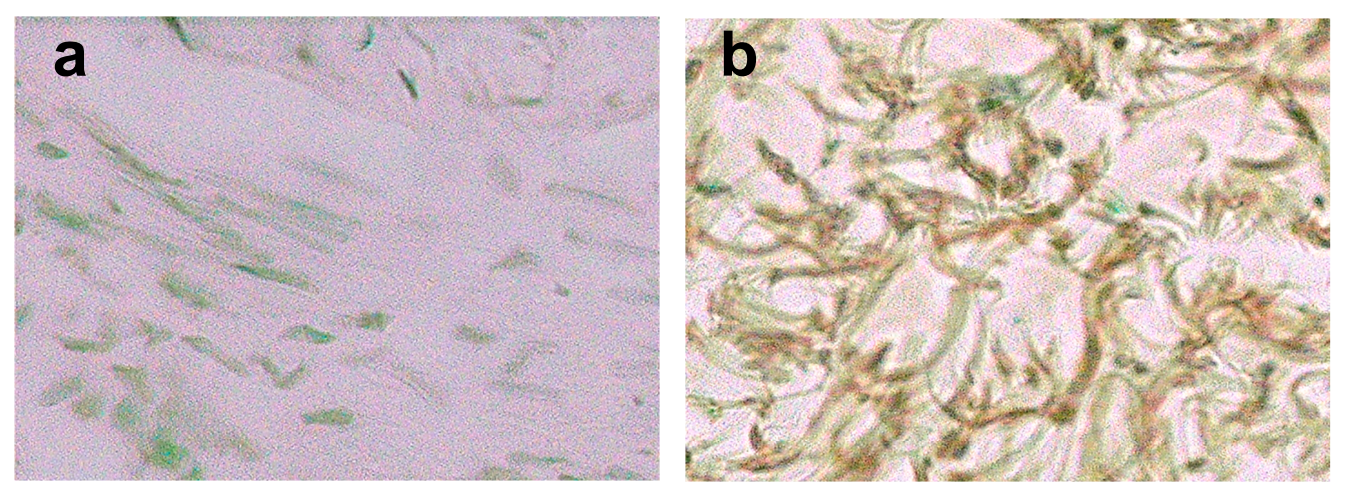

Figure 2

Yanaba et al. 


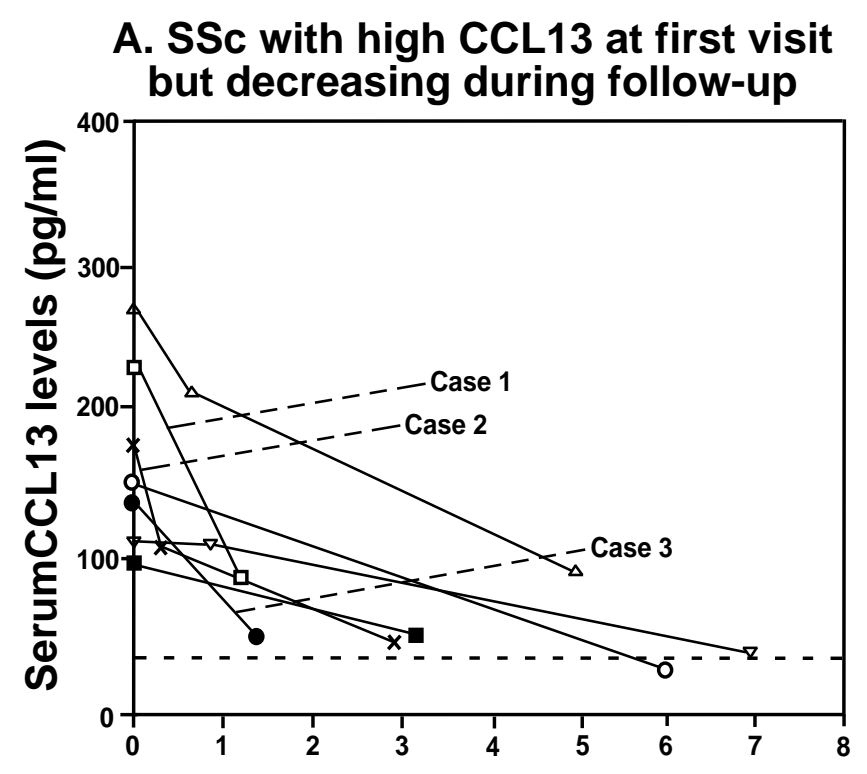

B. SSc with high CCL13 at first visit and stable during follow-up

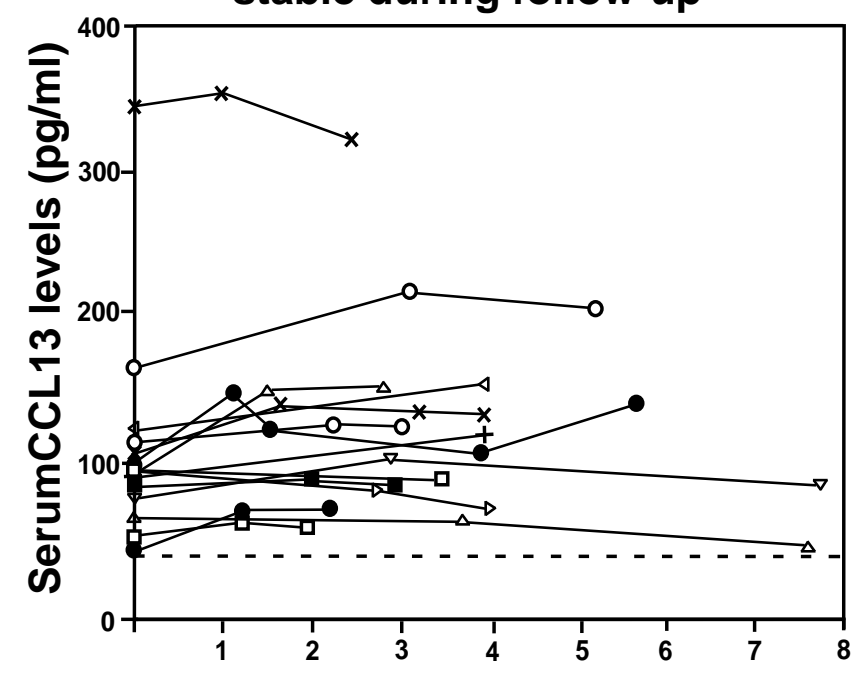

C. SSc with normal CCL13 at first visit

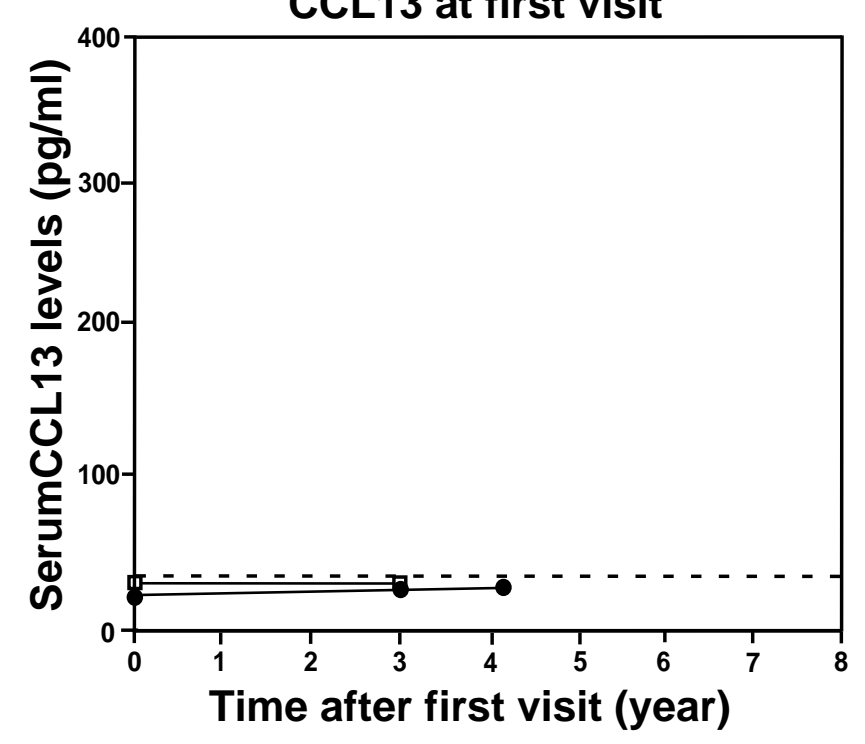

Figure 3

Yanaba et al. 
Table 1. Clinical and laboratory of in patients with SSc showing elevated serum CCL13 levels.

\begin{tabular}{lcc}
\hline & $\begin{array}{c}\text { Elevated CCL13 } \\
\mathrm{n}=76\end{array}$ & $\begin{array}{c}\text { Normal CCL13 } \\
\mathrm{n}=4\end{array}$ \\
\hline Age at onset, yrs, mean \pm SD & $47 \pm 15$ & $37 \pm 14$ \\
Male : Female & $11: 65$ & $1: 3$ \\
Duration, yrs, mean \pm SD & $4.2 \pm 5.7$ & $4.9 \pm 3.7$ \\
TSS, points, mean \pm SD & $13.4 \pm 9.3$ & $12.3 \pm 7.9$ \\
Clinical features \% & & \\
dSSc & 61 & 50 \\
1SSc & 39 & 50 \\
Pitting scars/ulcers & 30 & 50 \\
Contracture of phalanges & 41 & 0 \\
Diffuse pigmentation & 46 & 75 \\
Telangiectasia & 38 & 75 \\
Organ involvement \% & & \\
Pulmonary fibrosis & 58 & 25 \\
$\quad$ Decreased \%VC & 23 & 50 \\
$\quad$ Decreased \%DLco & 60 & 0 \\
Pulmonary hypertension & 15 & 50 \\
Esophagus & 69 & 0 \\
Heart & 11 & 0 \\
Kidney & 2 & 50 \\
Joint & 14 & 0 \\
Muscle & 11 & 25 \\
Laboratory findings \% & & 25 \\
Anti-topoisomerase I Ab & 42 & 25 \\
Anticentromere Ab & 37 & 25 \\
Increased IgG & 25 & \\
Elevated ESR & 47 & \\
Elevated CRP & 17 & \\
\hline & & \\
\hline
\end{tabular}

ESR, erythrocyte sedimentation rate; CRP, C-reactive protein.

Unless noted otherwise, values are percentages. Patient numbers are given in parentheses.

$* \mathrm{p}<0.05, * * \mathrm{p}<0.01$ versus SSc patients with normal serum CCL13 levels. 